\title{
Research on face recognition method of Airport security with Certificate Inspection
}

\author{
Zhang Ning ${ }^{1,2, a}$, Zhu Jin-fu',a \\ ${ }^{1}$ Guangzhou Civil Aviation College, Guangzhou, 510403, China \\ ${ }^{2}$ College of Civil Aviation of Nanjing University of Aeronautics \& Astronautics, Nanjing, 211100, \\ China \\ aanlan1008@126.com, bdaidai@nuaa.edu.cn
}

Keywords: airport; certificates inspection; relevant probability

\begin{abstract}
In the civil aviation airport, how to ensure the security of passenger travel is the focus of the work. Therefore, we need to inspect the certificates of passenger for travel security, andthe face recognition method is researched. An improved a face recognition method and inspection of certificate method are proposed for passenger travel security based on relevant rulesalgorithm. The facial features of people need to be inspected are extracted, and the relevantprobability of facial features are calculated. The disadvantages of traditional algorithm are overcome. The experimental results show that can improve the airport security certificate inspection efficiency, the face recognition efficiency is improved, and the satisfying results are obtained.
\end{abstract}

\section{Introduction}

With the rapid development of computer image recognition technology,face recognition of civil aviation airport security inspection has been obtained more and more attention from $p$ eople. To improve the recognition ability, and ensure the airport security, the face recogniti on algorithm is an important content in the field of civil aviation airport security research. The certificates inspection method of passenger is taken based on the face recognition[1-3], so it has become the core problem to research the civil aviation security. It plays an irrepl aceable role in the aviation airport security. At present, the civil aviation airport security in spection methods of certificates contain face recognition method of nonlinear dynamic transf ormation algorithm, the face recognition method based on genetic algorithm and the face re cognition method based on neural network algorithm. Among them, the most commonly use $\mathrm{d}$ algorithm is the face recognition method based on nonlinear dynamic transformation algor ithm. Due to the civil aviation airport security certificates inspection technology with face $r$ ecognition is very important, the applications is very extensive[4-7]. A lot of scholars have paid attention to it, and it has become a hot research topic in the area of civil aviation sec urity protection.

\section{Principle of face recognition method of civil airport security certificate inspection}

Civil aviation airport security certificate inspection method is taken based on face recognition, it is key issue in the research of civil aviation security protection. The traditional algorithm uses the object face recognition method, it cannot avoid the defects inspection objects recognition efficiency is low, and the quality of face images recognition is poor, the accurate rate of face recognition is reduced. Therefore, a face recognition method of civil aviation airport security certificate inspection is proposed based on relevant rules algorithm

\section{A Face feature extraction}

In accordance with the face image gray distribution feature of civil aviation airport security, the relevant state of face image is obtained. . Because the face feature has some relevance features, and therefore this correlation relevance features can be used as the face recognition feature in the process of civil aviation airport security certificate detection. The relevant features are applied in 
the face recognition.

Set $h(z, a)$ is the collected face images of civil aviation airport security objects, and the area of 2D image is $P \times R$, in the specified location, the spatial location of the face area selection at any point is $\left(a_{1}, a_{2}\right)$, the spatial location selected based on the human body is $\left(b_{1}, b_{2}\right)$, he two regions selected points are used for the deflection angle determination, in connection with the horizontal direction of the two regions, the deflection angle in connection with the horizontal direction of the two regions selected points is $\alpha$, The probability of two points belongs to the face recognition state interval is selected by the following formula:

$$
\begin{aligned}
& R(k, l \mid \alpha)=\gamma\left\{\left(a_{1}, a_{2}\right),\left(b_{1}, b_{2}\right) \in\right. \\
& \left.P \times R \mid \alpha, h\left(a_{1}, a_{2}\right)\right\}
\end{aligned}
$$

Where, $\gamma(a)$ is used to describe the number of face feature extraction. $\alpha$ is used to describe the position of human face characteristic space, the selected point of contact is calculated, and the facial feature parameters are calculated by:

$$
\left\{\begin{array}{c}
X=(z-a-f)[\min (z, a, f)] \\
M=2(z-a-f)^{2}
\end{array}\right.
$$

In the above formula, $X$ is the feature parameter of face image.

According to the above method, it can extract the civil aviation airport security certificate inspection personnel face feature, the space position the distribution of these characteristics is shown in Figure 1.

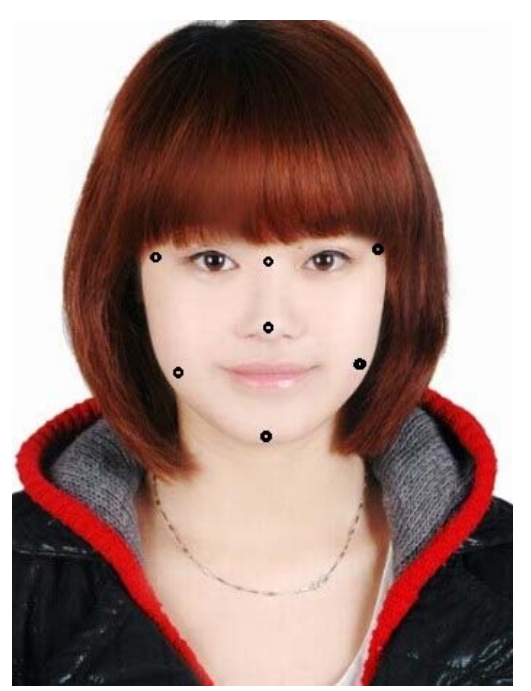

Figure 1 Location distribution of space features

According to the method described above, the characteristic parameters of face are obtained, the parameters are used to describe the civil aviation airport security inspection documents, according to the changes of these properties, it can describe the dynamic change situation of human face, and it lays the accurate data base for face recognition.

\section{B Relevant probability calculation of facial feature}

The relevance the probability of facial features the civil aviation airport security check c ertificates is calculated, dynamic change relationship between different facial features are co mbined together. The face of the personnel consistent with the security certificate, the face recognition and civil aviation airport security are obtained.

Sets the number of all the features extracted from face images is $q$, the $l_{\text {th }}$ face featur e is $f_{l}$. All the facial features can be divided into different $M$ categories, the facial feature subset is $\left(X_{1}, X_{2}, \cdots, X_{p}\right)$, all the facial feature subset is composed of facial features $f_{l}=\left(Y_{l 1}, Y_{l 2}, \ldots ., Y_{l q}\right)$, where $l=0,1, \cdots, q$. Facial feature correlation method is used to extract 
the different feature subsets for classification, using the following formula for extract the se curity certificate personnel characteristics $g_{l}$ :

$$
q\left(g_{l} \cdot d_{k}\right)=\left(q\left(g_{l}\right)+q\left(f_{l} \cdot \delta_{w}\right)\right) / q\left(d_{k}\right) \quad l=0,1, \cdots, q
$$

Wherein, the $q\left(d_{k}\right)$ is the prior probability of face recognition with civil aviation airport security certificates detection, and $q\left(g_{l} \cdot d_{k}\right)$ is the priori conditional probability of facial fe ature classification. For the facial features of the same type, recognition result of $q\left(g_{l}\right)$ are not changed, use the following formula to calculate the probability of relevance between $t$ he same species in a data set of facial features:

$$
\begin{aligned}
& q\left(g_{l} \cdot d_{k}\right)=q\left(x_{1} \cdot d_{k}\right)+q\left(x_{2} \cdot d_{j}\right)+\cdots \\
& +q\left(x_{n} \cdot d_{k}\right)=\sum_{l=2} q\left(x_{l} \cdot d_{k}\right) \\
& q\left(d_{k}\right)=\sum_{l=2}^{q} q\left(g_{l}\right) / q\left(d_{k} \cdot g_{l}\right)
\end{aligned}
$$

The probability of relevance feature of the facial feature is calculated in the following e quation:

$$
q\left(d_{k} \cdot g_{l}\right)=U\left(p\left(d_{k}\right)+g_{l}\right) / U_{f l}^{2}
$$

In the above formula, $U\left(p\left(d_{k}\right)+g_{l}\right)$ is the number of features facial feature data of sub categories $g_{l}$ in the presence of concentrated features, $T_{g l}$ is the number of features in the face image recognition. Assuming the relevance probability value is greater than 0.8 , deter mine the face and the civil aviation airport security documents inspection object, and the $o$ bjects is consistent. Otherwise, the face and the civil aviation airport security are inconsiste nt. On the basis of the method of the above described, facial features are extracted. The fa cial feature relevance probability is obtained, so as to complete the face recognition and the civil aviation airport security inspection.

\section{Simulation results}

In order to verify the validity of this algorithm, an experiment is taken. 100 pieces of civil aviation airport security documents object images are collected, the traditional algorithm and the new method are used in the experiment for comparison, the face recognition is simulated. The related parameter are set as follows: $t_{i}=38, n=100, h_{i}=48, u=49, U=85, \eta_{i}=31$, the face recognition results are calibrated, and the specific results are shown in Figure 2.

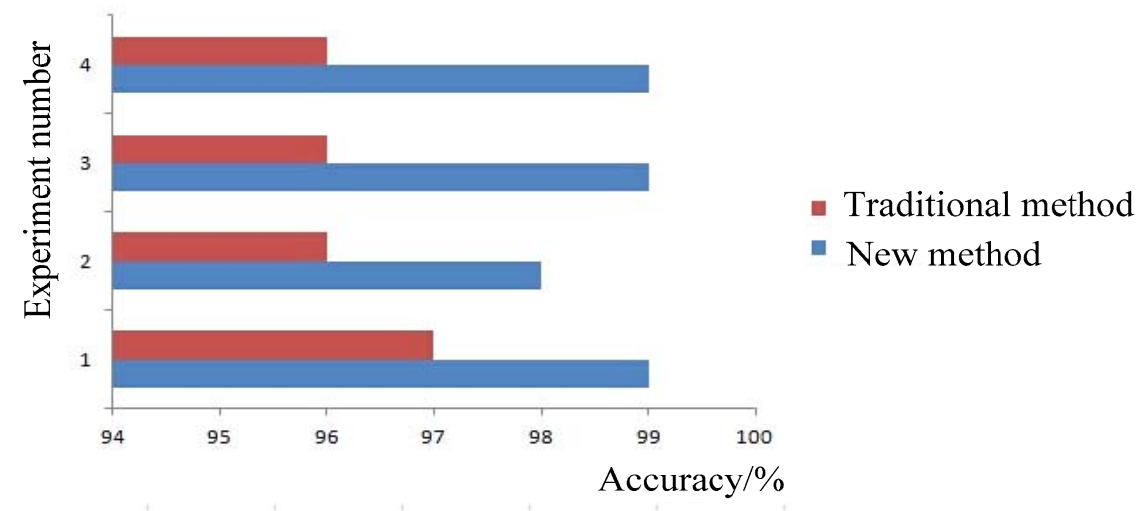

Figure 2. Face recognition results of different algorithms

In the figure above, the civil aviation airport security certificates are recognized, and the recognition rate of the new method is higher than traditional method, the algorithm can be applied in the inspection of certificates in airport, when the face image quality is worse, face recognition has 
certain superiority.

The relevant data in the experiment are collected, and the results are recorded in Tables 1 and 2. Table 1 is the related data of face recognition when the inspection object face image quality is better, and table 2 is the recognition result when the object face image quality is worse.

Table 1 Recognition data when the image quality is good

\begin{tabular}{ccc}
\hline Recognition data & Traditional algorithm & New algorithm \\
\hline The number of image recognition & 100 & 100 \\
Recognition times of inspection objects & 98 & 98 \\
Actual number of inspection & 98 & 98 \\
Recognition error & $0 \%$ & $0 \%$ \\
\hline Table 2 Recognition data & when the image quality is & bad \\
\hline Recognition data & Traditional algorithm & New algorithm \\
\hline The number of image recognition & 100 & 100 \\
Recognition times of inspection objects & 97 & 98 \\
Actual number of inspection & 98 & 98 \\
Recognition error & $1 \%$ & $0 \%$ \\
\hline
\end{tabular}

From the results, we can conclude the new method is better than the traditional method, the experimental results show that can improve the airport security certificate inspection efficiency, the face recognition efficiency is improved, and the satisfying results are obtained. The accuracy rate of object is much higher than traditional algorithm in face recognition.

\section{Conclusions}

In this paper, an improved a face recognition method and inspection of certificate method are proposed for passenger travel security based on relevant rules algorithm. The facial features of people need to be inspected are extracted, and the relevant probability of facial features are calculated. The disadvantages of traditional algorithm are overcome. The experimental results show that can improve the airport security certificate inspection efficiency, the face recognition efficiency is improved, and the satisfying results are obtained. It can meet the actual demand of airport security, it can ensure the passenger safety.

\section{References}

[1] XU Xiao-bin, ZHANG Guang-wei, SUN Qi-bo, et al. Precision Configurable Data Aggregation Algorithm in WSNs[J]. ACTA ELECT R ONICA SINICA, 2014, 42(6):1205-1209.

[2] ZHENG Zhen, WANG Liyuan, ZHOU Yong. Chaos Theory and Its Application in Ships Target Recognition[J].Ship Electronic Engineering, 2013, 33(5): 48-50.

[3] GUO Rong-yan, HU Xue-hui. Study aboutLicense Plate Recognition Based on Back Propagation Neural Network[J]. Computer Simulatio, 2010,27(9): 299-301.

[4] QIU Bao-zhi, CHEN Xu. Border Image Segmentation Technology for Serious Collision Vehicle in Accident[J]. Computer Simulation, 2013, 30(1): 215-218.

[5] Zhu Zhen. A Support Vector Machine Algorithm Based on Pretreatment of Neural Network Ensemble[J]. Bulletin of Science and Technology, 2013,29(4): 26-30.

[6] SUI Xin. Study and Implementation on Host Characteristic Information Passively Identifying[J]. Science Technology and Engineering, 2013, 13(3): 652-658.

[7] Kara L B,Stahovich $\mathrm{T}$ F. An image-based,trainable symbol recognizer for hand-drawn sketches[J].Computers and Graphics,2005,(04):501-517. 